\title{
La tutoría virtual, un modelo alternativo para mejorar la eficiencia terminal en el nivel superior
}

\section{Virtual tutoring, an alternative terminal model to improve efficiency in the upper level}

\author{
DOI: $10.46814 /$ lajdv3n6-012
}

Recebimento dos originais: 01/10/2021

Aceitação para publicação: 24/11/2021

\section{Tania Torres Vázquez}

Doctorando en Educación, IESPE.

Universidad para el Bienestar Benito Juárez García, sede Francisco I. Madero, Hidalgo. Miguel Miramón Mza.18, lote 3, Colonia Militar, Mineral de la Reforma Hidalgo, México.

E-mail: tanis_7907@hotmail.com

\section{RESUMEN}

La presente comunicación, trata sobre la tutoría virtual y de cómo este modelo puede influir de manera positiva en la eficiencia terminal en el nivel universitario, cabe señalar, que este es sólo el inicio de la investigación (estado del arte y/o conocimiento) que guarda el tema y que la metodología, resultados y conclusiones corresponden exactamente a ese apartado. Por lo tanto se analizan los modelos de tutoría: presencial y virtual, que se llevan a cabo en la Universidad Autónoma del Estado de Hidalgo (UAEH), México, con el objetivo es llevar a cabo una evaluación de ambos modelos para así poder estructurar una propuesta acode a las necesidades actuales de la universidad. Dicha propuesta plantea la utilización de un modelo de tutoría diferente, al que se ha estado trabajando en la UAEH, este modelo es el de la tutoría presencial, donde el tutorado asiste a una entrevista cara a cara con su tutor, se debe mencionar que aunque este modelo funciono muy bien en sus inicios, hoy día nos enfrentamos a la globalización y a la sociedad del conocimiento que nos brindan nuevas herramientas, las cuales debemos utilizar, si nos ofrecen la oportunidad de mejorar y apoyar a más estudiantes, mismos que son parte de la llamada generación net, precisamente porque utilizan las Tecnología de la Información y Comunicación de forma cotidiana, por lo que se considera conveniente emplear un modelo de Tutoría Virtual, el cual implique foros, chats, páginas web, donde los alumnos puedan hablar sobre diferentes problemáticas.

Palabras clave: orientación, tutoría virtual, educación superior, retos.

\begin{abstract}
This communication deals with virtual mentoring and how this model can positively influence the completion rate at the college level, it should be noted that this is only the beginning of the investigation (state of the art and / or knowledge) that save the topic and methodology, results and conclusions correspond exactly to that section. Therefore models are analyzed tutoring, classroom and virtual, that are conducted at the Autonomous University of Hidalgo State (UAEH), Mexico, with the aim is to carry out an evaluation of both models in order to structure a acode to the current needs of the proposed university. This proposal suggests the use of a different model of mentoring, which has been working on UAEH, this model is the face mentoring, tutoring where attending a face to face interview with your tutor, it should be mentioned that although this model worked well in the beginning, today we face globalization and the knowledge society that give us new tools, which we use, if we provide the opportunity to improve and provide more support for students who are part thereof call net generation,
\end{abstract}


precisely because they use the Information Technology and Communication on a daily basis, so it is considered appropriate to use a model of Virtual Tutoring, which involves forums, chats, websites, where students can talk about different problematic

Keywords: orientation, virtual mentoring, higher education challenges.

\section{INTRODUCCIÓN}

La Globalización, producto del liberalismo económico, es decir, el libre mercado, la internacionalización o como la llaman algunos otros mundialización y la Sociedad del Conocimiento, han impuesto una nueva agenda para las universidades, que viven hoy grandes cambios producto de las políticas educativas que las naciones implementan para crecer al ritmo que la modernidad les compromete. Ya que, como lo menciona Berger, la ubicación en la sociedad es a través de coordenadas asignadas de manera externa, (Berger L. , 1977) en nuestro caso las coordenadas están dictadas por esas pequeñas "elites", las cuales dice Mills, están formada por los que tienen el mando en las instituciones directivas, y que por lo tanto tienen la capacidad de influir en la vida de otros hombres o mujeres "ordinarios" (Mills, Wright, 1971), es decir los organismos nacionales (ANUIES) e internacionales (OCDE).

$\mathrm{Y}$ es precisamente en este contexto y bajo estas circunstancias que surge el Programa Institucional de Tutoría, cuyo objetivo es:

Proporcionar al estudiante un tutor que le acompañe, guie y oriente, durante su trayectoria universitaria para contribuir en su desarrollo integral mediante el otorgamiento de servicios de: tutorías, asesorías académicas, atención psicológica, atención médica, orientación vocacional y escolar, becas internas y externas, así como acciones preventivas universitarias de salud, la cobertura en la afiliación al seguro facultativo del IMSS y la difusión de la oferta educativa, fortaleciendo su desarrollo integral (UAEH, 2007).

El PIT se ha implementado en diferentes universidades a petición de la Asociación Nacional de Universidades e Instituciones de Educación Superior (ANUIES), por su parte en la UAEH, se han hecho los esfuerzos pertinentes para contar con el programa un poco antes de la petición de ANUIES, actualmente cada instituto, escuela superior, escuela preparatoria y el SUV, cuentan con el PIT.

El objetivo de dicho programa es proporcionar acompañamiento, guía y orientación a los estudiantes. Se observa que la UAEH, siempre atenta a las necesidades y pendiente del contexto local, regional, nacional e internacional en el que se desenvuelven los estudiantes, ha implementado diferentes modelos de tutoría, no sólo presencial, sino también virtual, lo cual es el motivo del análisis del presente documento. 


\section{MARCO TEÓRICO Y OBJETO DE ESTUDIO}

Para la UAEH proporcionar acompañamiento siempre ha sido muy importante, más allá de que los organismos como la ANUIES y OCDE se lo recomienden, ¿por qué se afirma esto? Las OCDE realizó "Exámenes de las políticas nacionales de educación en México" (1994), y a partir de ello recomendó desarrollar los servicios de tutoría y de apoyo a la orientación de los escolares y estudiantes de los niveles medio superior y superior ; por su parte, ANUIES realizó un análisis profundo respecto a la situación actual de las Instituciones de Educación Superior (IES) y de las expectativas que tienen hasta 2020, el proceso duro más de un año, desde mediados de 1998 hasta octubre de 1999, e implicó el trabajo de un grupo técnico de la Secretaría General Ejecutiva de la ANUIES y una amplia participación de las instituciones afiliadas. De aquí surgen 14 programas de acción, de los cuales el de Desarrollo integral de los alumnos, es de alto interés, ya que plantea lo siguiente:

Apoyar a los alumnos del SES, a través del diseño e implementación por las IES de programas de desarrollo integral, de suerte que una elevada proporción de ellos culmine sus estudios en el plazo previsto y logre los objetivos de formación establecidos en los planes y programas de estudio (ANUIES, 1999).

Se observa que los períodos de trabajo son 1994-1999, sin embargo, el anteproyecto de tutoría de la UAEH data de 1992, donde está ya contaba con tres modalidades: individual, grupal y por pares; actualmente se utilizan la individual, grupal y virtual (sólo en las carreras del Sistema de Universidad Virtual SUV, es decir, educación a distancia), por lo que es la Tutoría, emanada de este Programa, el objeto de estudio, ¿por qué? pues se considera que el modelo virtual, aplicado a la educación presencial puede mejorar la eficiencia terminal en el nivel superior.

\subsection{LA IMPORTANCIA DE EMPLEAR EL MODELO DE TUTORÍA VIRTUAL}

Ahora bien, los modelos presencial y virtual, se han implementado en diferentes contextos, por un lado el primero en la educación escolarizada o presencial, que se entiende como la educación que se recibe diariamente en las aulas de los diferentes institutos o escuelas superiores de la UAEH y el último en la educación a distancia, que es la que se encarga de impartir el SUV, a través de las diferentes Tecnologías de la Información y Comunicación, sin embargo, se considera que, en la educación escolarizada también se puede utilizar la tutoría virtual, partiendo de lo siguiente: la sociedad del conocimiento y la globalización de la que hablábamos en un principio, exigen ciudadanos con nuevas habilidades, capaces de utilizar los conocimientos en la resolución de problemas de la vida cotidiana, personas que sean capaces de identificar la información que vale la pena de la que no, que sean autónomos y autodidácticas, para que pueda existir una verdadera competencia, esto implica, utilizar todos los recursos que estén a nuestro alcance para lograrlo, y en este sentido la Tecnologías de la 
Información y la Comunicación (TIC), brindan un gran apoyo para desarrollar en los estudiantes del nivel superior las habilidades antes mencionadas, a través de tutores que se encarguen de dar seguimiento a los alumnos y sus procesos tanto de enseñanza aprendizaje como los familiares, institucionales y administrativos.

El sustento teórico de la tutoría en este trabajo se obtiene de la Teoría de los campos de Pierre Bourdieu, ya que para él un campo es: "espacios de juego históricamente constituidos con sus instituciones específicas y sus leyes de funcionamiento propias" (Bourdieu, 1987: 108), es decir, el Programa de Tutorías es un campo, puesto que se ha constituido a través del tiempo y posee leyes de funcionamiento propias, contempladas en el Programa Institucional de Tutoría.

En este orden de ideas, los campos producen prácticas determinadas, es decir, son personas que piensan y actúan de acuerdo a sus habitus, producto de la internalización de lo que la sociedad les demanda (BOURDIEU, 2005).

Todo lo anterior determina los vínculos que han creado para ser parte de este campo y las dificultades que han enfrentado para lograrlo, este es el punto más importante, ya que la tutoría vendría a ser uno de esos vínculos o relaciones, tal como lo expresa Barraul (2007) " el establecimiento de vínculos denota la pertenencia a un espacio común construido, significado y a fín de cuenta inventado" (p.161), luego entonces pueden favorecer el sentimiento de pertenencia de los alumnos universitarios y contribuir a que estos no abandonen la escuela, y de esa forma elevar el índice de Eficiencia Terminal, o lo que para Uriz (1994) es el establecimiento de la relación vital entre el sujeto y su entorno (p. 37).

\section{HACÍA UN ENFOQUE CUALITATIVO}

Se utilizó una metodología de corte cualitativo, puede definirse como un conjunto de prácticas aclarativas que hacen visible lo que antes no era considerado importante, lo transforman y convierten en una serie de representaciones a partir de observaciones, anotaciones, grabaciones y documentos. Es comprensivo, interpretativo y en cierta medida crítico, por lo que al final de la investigación se pretende modificar la eficiencia terminal, es decir, incidir sobre el desempeño académico (para prevenir la deserción escolar) y elevar los índices de Eficiencia Terminal (E.T) a través de la adopción del modelo de Tutoría Virtual

La investigación cualitativa permite explicar más ampliamente los datos, contextualizar la situación. Se ha ampliado más en las disciplinas humanísticas por lo que se considera el más adecuado para la presente investigación.

El alcance será exploratorio debido a que en este momento lo que se busca es conocer a fondo cada una de las modalidades de Tutoría que se utilizan en la UAEH, compararlos entre sí y de ser posible verificar el impacto que cada uno ha tenido. 
Así, se realizó una primera búsqueda de las modalidades de tutoría en la UAEH. Posteriormente, se realizó un análisis de contenido formándose categorías de análisis a partir de las similitudes o divergencias de la información (Marcelo, 1989), lo que permitió identificar las modalidades de tutoría predominantes en la UAEH. Cabe señalar que se presenta una versión sintetizada de los resultados.

\section{LOS HALLAZGOS}

Como ya se mencionó, los datos aquí registrados son el producto de la búsqueda ejecutada para formalizar el estado de la cuestión o del conocimiento que guarda el Modelo de Tutoría Virtual en la UAEH, y de ahí poder establecer la viabilidad y pertinencia de un proyecto de un investigación, que versará sobre este modelo y la forma en que el mismo puede contribuir al aumento de la Eficiencia Terminal en el nivel superior, es decir, en la universidad.

Tabla 1. Modelos de Tutoría

\begin{tabular}{|l|l|}
\hline PRESENCIAL & A DISTANCIA/VIRTUAL \\
\hline $\begin{array}{l}\text { La tutoría es un proceso de acompañamiento } \\
\text { de tipo personal y académico a lo largo del } \\
\text { proceso formativo para mejorar el } \\
\text { rendimiento académico, solucionar } \\
\text { problemas escolares, desarrollar hábitos de } \\
\text { estudio, trabajo, reflexión y convivencia } \\
\text { escolar (González Cano, García González \& } \\
\text { Hernández García, 2011) }\end{array}$ & $\begin{array}{l}\text { Latoría virtual, es una actividad docente } \\
\text { distancia y en contenidos, o solo en educación } \\
\text { materiales didácticos digitales, promoviendo } \\
\text { la interacción con y entre los estudiantes a } \\
\text { través de medios tecnológicos, para } \\
\text { motivarlos al logro de los objetivos } \\
\text { educativos. (UAEH, s/f) }\end{array}$ \\
\hline
\end{tabular}

INDIVIDUAL: Es la que se le brinda al alumno de manera personalizada, en entrevista tutorado-tutor, o a distancia.

GRUPAL: Es la que brinda el tutor a un grupo de alumnos. Para llevarla a cabo se requiere de una planeación previa. Parte importante, es que incluye la tutoría entre pares, es decir, donde los alumnos se brindan acompañamiento mutuamente.

La tutoría es un proceso de acompañamiento de tipo personal y académico a lo largo del proceso formativo para mejorar el rendimiento académico, solucionar problemas escolares, desarrollar hábitos de estudio, trabajo, reflexión y convivencia escolar (González Cano, García González \& Hernández García, 2011) La tutoría virtual, es una actividad docente que realiza un experto en enseñanza a distancia y en contenidos, o solo en educación a distancia, para guiar el aprendizaje, orientar y facilitar la utilización de recursos y materiales didácticos digitales, promoviendo la interacción con y entre los estudiantes a través de medios tecnológicos, para motivarlos al logro de los objetivos educativos. (UAEH, s/f)

INDIVIDUAL: Es la que se le brinda al alumno de manera personalizada, en entrevista tutorado-tutor, o a distancia. 
GRUPAL: Es la que brinda el tutor a un grupo de alumnos. Para llevarla a cabo se requiere de una planeación previa. Parte importante, es que incluye la tutoría entre pares, es decir, donde los alumnos se brindan acompañamiento mutuamente.

\subsection{LA TUTORÍA PRESENCIAL}

Como se indicó anteriormente, la Tutoría Presencial, es un proceso de acompañamiento, lo que la diferencia de la Tutoría a Distancia es que esta se da cara a cara, tutor-tutorado, en un lugar establecido por la institución para este propósito. Esta puede ser individual o grupal.

\subsection{LA TUTORÍA A DISTANCIA/VIRTUAL}

La Tutoría a Distancia, por su parte, es una actividad docente, encaminada a guiar, orientar y facilitar la utilización de recursos y materiales didácticos digitales, además de promover la interacción con y entre los estudiantes a través de medios tecnológicos, esta última parte es la que la hace diferente a la Tutoría Presencial, ya que también se brinda de manera individual y grupal.

Ahora bien, la UAEH, considera al estudiante como el centro de todos sus esfuerzos, así se reconoce que no existe un tipo único de estudiante, cada uno es un sujeto, con una historia de vida y un contexto particular, a pesar de que todos comparten una identidad como estudiantes de la UAEH, por tanto para el SUV, no existe un sólo modelo de Tutoría Virtual, sino varios.

Existen cuatro modelos con los que se trabaja en el SUV de la UAEH: A, B, C y D. en cada modelo existen funciones académica, pedagógica, organizativa, institucional, tecnológica y motivacional.

Las cuales explicaremos a grandes rasgos para una mejor comprensión de los modelos.

Académica: Asesorar en relación con los contenidos. Moderar discusiones para la construcción del conocimiento. Revisar y evaluar las actividades de aprendizajes.

Pedagógica: Proporcionar estrategias de aprendizaje, así como recomendaciones y monitorear la elaboración y calidad de trabajos. Realizar un seguimiento para asegurarse que los estudiantes trabajan a un ritmo adecuado para prever la deserción.

Tecnológica: Asesorar en el manejo de herramientas de comunicación de la plataforma educativa; en la descarga de materiales educativos y en la selección y uso de software educativo para el desarrollo de las actividades de aprendizaje.

Motivacional: Acompañar y dinamizar la acción formativa, motivar para el trabajo efectivo y oportuno del estudiante. Animar y estimular la participación. Realizar la motivación al esfuerzo y logros e identificar dando atención de problemáticas surgidas en la interacción social. 
Institucional: Atender y/o canaliza inquietudes de tipo administrativo sobre la inscripción, permanencia y egreso (certificación, diploma, grado...) Ser un enlace con la coordinación del programa para realizar la trayectoria académica y evaluación del proceso educativo, así como investigación e innovación educativa.

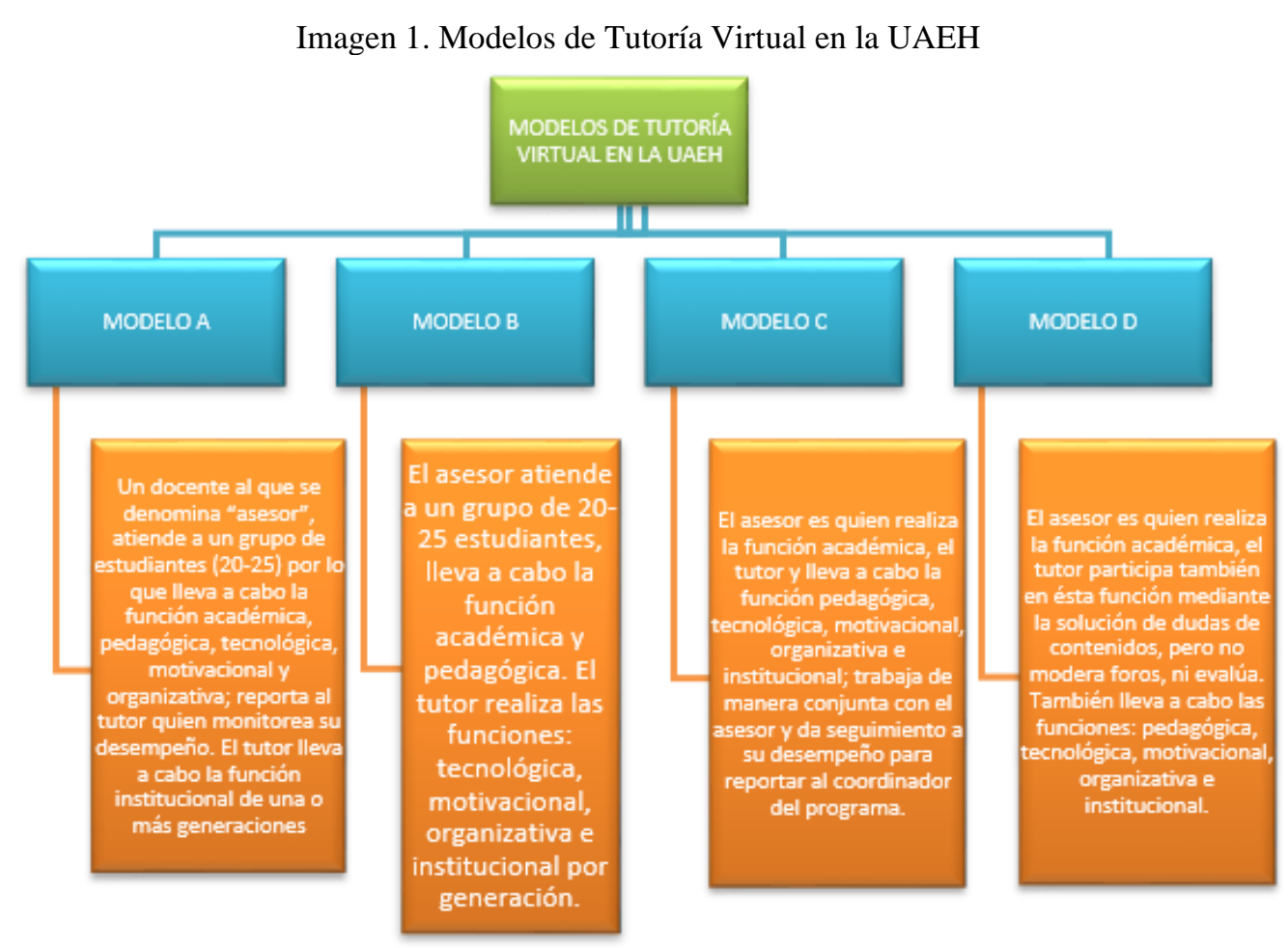

Se observa que cada modelo cuenta con dos figuras, el asesor y el tutor, el asesor es quien se encarga principalmente de la función académica y el tutor de la función tecnológica, motivacional, organizativa e institucional.

En el caso del modelo A, el tutor sólo lleva a cabo la función institucional, mientras que en el modelo B y C lleva a cabo la función tecnológica, motivacional, organizativa e institucional y en el caso del modelo D, si comparte parte de la función pedagógica.

\section{A MANERA DE CONCLUSIÓN}

La UAEH, dentro de todos sus institutos, escuelas superiores y preparatorias ha implementado el Programa Institucional de Tutoría, con la finalidad de brindar acompañamiento a los estudiantes, en todos los semestres, no obstante, dadas las nuevas condiciones, ha tenido que agregar un modelo nuevo que es el de la tutoría virtual, que por el momento sólo opera en el SUV, sin embargo se reconoce la importancia y urgencia de implementar dicho modelo en la educación presencial y no sólo en la educación a distancia. 
¿Por qué se propone esto? Debido a que los alumnos que actualmente cursan una licenciatura, son los llamados "nativos digitales" que pertenecen a la generación NET, ¿qué significa esto? "son los actuales jóvenes, adolescentes y niños, nacidos entre 1980 y 2000. Crecieron en la era de la informática y la inmediatez" (MASCLEF, Medina Galván, \& Usandivaras, 2010) .

No sólo son parte de una nueva generación de estudiantes, sino de una nueva cultura en la que trasladan su pensamiento y relaciones sociales al campo de las nuevas tecnologías de la información y la comunicación (TIC's), estas les resultan imprescindibles en sus labores diarias, poseen grandes habilidades para jugar, interactuar, buscar y resolver mediante estrategias casi innatas de aprendizaje; son capaces de elaborar y desarrollar proyectos conjuntos sin importar la distancia ni el tiempo (BAEZ Ornelas, 2008) . Quieren todo ya y les aburren las reuniones. Aprenden haciendo. Esta generación está formada por personas que tienen en su cotidianidad recursos tecnológicos de comunicación y telemática a su alcance" (MASCLEF, et al, 2010)

Por lo tanto son jóvenes que usan la tecnología a todas horas y para quienes es más fácil entrar a internet para realizar diferentes actividades, tener la libertad de elegir el tiempo en el que deben realizar la actividad a través de su medio favorito (teléfono o computadora) puede ser de gran utilidad para que no se resistan a llevar a cabo la tutoría y a la vez poder encontrar material que le sea de utilidad para resolver sus dudas en el momento, son ventajas de la tutoría virtual, la cual también supone una ventaja para el tutor, pues si se alimenta bien la base de datos, este se ahorra tiempo y esfuerzo en las cuestiones administrativas (sobre todo de llenado de formatos).

Se observar que estos sujetos tienen características muy especiales las cuales contribuyen a que construyan su conocimiento de manera diferente y también que actúen diferenciadamente, en este sentido es como cobran importancia las palabras de Berger y Luckmann (2012) "el yo no puede entenderse adecuadamente si se los separa del contexto social particular en el que se formaron”, de ahí la importancia de tomar en cuenta al sujeto como tal, y también su contexto.

Tomando en cuenta estas características, tanto del contexto como de los sujetos, es que se propone el modelo virtual como una alternativa para mejorar la Eficiencia Terminal del nivel superior (universidad). 


\section{BIBLIOGRAFÍA}

ANUIES. (2002). Programa Institucional de tutoría. México: ANUIES.

BAEZ Ornelas, D. (2008). Generación net. Docentes innovando, 1-2.

BARRAULT, O. (2007). Los espacios de encuentro en la psicología comunitaria y sus implicaciones en la subjetividad. Revista de Ciencias Humanas UTP, 155-167.

BERGER, P. (2009). Introducción a la sociología. Barcelona: Península.

Berger, P. L., \& Luckmann, T. (2012). La construcción social de la realidad. Buenos Aires, Argentina: Amorrortu.

BOURDIEU, P. (1987). El interés del sociólogo, cosas dichas. Buenos Aires: Gedisa, pp. 108-114.

BOURDIEU, P. (2005). Una invitación a la sociología reflexiva. México: Siglo XXI.

González Cano, M., García González, C., \& Hernández García, T. J. (2011). La tutoría como estrategia de apoyo para alumnos en riesgo de baja. 3er. Congreso Internacional de Orientación Educativa, Vocacional y Tutoría (págs. 1-15). Baja California, México: UBC.

GUTIERREZ, A. (2005). Las prácticas sociales: una introducción a Pierre Bourdieu. Córdoba: Ferreyra Editor.

MARDONES, J. (1991). Universidad Nacional Abierta. Recuperado el 05 de Agosto de 2014, de Filosofía de las Ciencias Humanas y Sociales. Nota histórica de una polémica incesante: postgrado.una.edu.ve/filosofia/paginas/mardones1.pd

MASCLEF, M. A., Medina Galván, M., \& Usandivaras, S. (2010). Educación Superior: buscando respuestas para la Generación Net. . X Coloquio Sobre Gestión Universitaria En América Del Sur. Instituto de Administración, Facultad de Ciencias Económicas (págs. 1-13). Mar de la Plata, Argentina: X Coloquio Sobre Gestión Universitaria En América Del Sur. Instituto de Administración, Facultad de Ciencias Económicas.

OCDE. (1997). Exámenes de las políticas nacionales de educación. México: OCDE.

UAEH. (2014). Universidad Autónoma del Estado de Hidalgo. Recuperado el 20 de Agosto de 2014, de Instituto de Ciencias Sociales y Humidades: http://www.uaeh.edu.mx/campus/icshu/ubicacion.htm

UAEH. (s.f.). Manual para el docente de educación a distancia. Recuperado el 02 de Julio de 2014, de Sistema de Universidad Virtual: www.uaeh.edu.mx/docencia/VI_Lectura/educ_continua/.../LECT56.pdf URIZ, J. (1994). La subjetividad de la organización. El poder más allá de las estructuras. México: Siglo XXI. 